\title{
Fetal dose from Head and Neck Tomotherapy Versus 3D Conformal Radiotherapy
}

\author{
So Hyun Park' , Won Hoon Choi ${ }^{2}$, Jinhyun Choi ${ }^{1}$ \\ ${ }^{1}$ Department of Radiation Oncology, Jeju National University Hospital, Jeju, Korea; ${ }^{2}$ Department of Radiation Oncology, Yonsei University College of \\ Medicine, Seoul, Korea
}

\section{Original Research}

Received August 6, 2019

Revision November 27, 2019

Accepted December 9, 2019

Corresponding author: Jinhyun Choi

Department of Radiation Oncology, Jeju National University Hospital,

15 Aran 13-gil, Jeju-si, Jeju-do 63241,

Korea

Tel: +82-64-717-1328

Fax: +82-64-717-1323

E-mail: radoncogene@gmail.com

This is an Open-Access article distributed under the terms of the Creative Commons Attribution NonCommercial License (http://creativecommons.org/ licenses/by-nc/3.0) which permits unrestricted noncommercial use, distribution, and reproduction in any medium, provided the original work is properly cited.

Copyright $\odot$ 2019The Korean Association for Radiation Protection

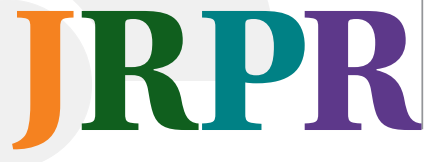

Background: To compare the dose of radiation received by the fetus in a pregnant patient irradiated for head and neck cancer using helical tomotherapy and three-dimensional conformal radiation therapy (3DCRT).

Materials and Methods: The patient was modeled with a humanoid phantom to mimic a gestation of 26 weeks. Radiotherapy with a total dose of $2 \mathrm{~Gy}$ was delivered with both tomotherapy ( 2.5 and $5.0 \mathrm{~cm}$ jaw size) and 3DCRT. The position of the fetus was predicted to be $45 \mathrm{~cm}$ from the field edge at the time of treatment. The delivered dose was measured according to the distance from the field edge and the fetus.

Results and Discussion: The accumulated dose to the fetus was $1.6 \mathrm{cGy}$ by 3DCRT and 2 and $2.3 \mathrm{cGy}$ by the 2.5 and $5 \mathrm{~cm}$ jaw tomotherapy plans. For tomotherapy, the fetal dose with the 2.5 $\mathrm{cm}$ jaw was lower than that with the $5 \mathrm{~cm}$ jaw, although the radiation leakage was greater for 2.5 $\mathrm{cm}$ jaw plan due to the 1.5 fold longer beam-on time. At the uterine fundus, tomotherapy with a $5 \mathrm{~cm}$ jaw delivered the highest dose of $2.4 \mathrm{cGy}$. When the fetus moves up to $35 \mathrm{~cm}$ at the 29th week of gestation, the resultant fetal doses for 3DCRT and tomotherapy with 2.5 and $5 \mathrm{~cm}$ jaws were estimated as $2.1,2.7$, and $3.9 \mathrm{cGy}$, respectively.

Conclusion: For tomotherapy, scattering radiation was more important due to the high monitor unit values. Therefore, selecting a smaller jaw size for tomotherapy may reduce the fetal dose. however, evaluation of risk should be individually performed for each patient.

Keywords: Tomotherapy, 3DCRT, Fetal Dose, Pregnant, Head and Neck Cancer

\section{Introduction}

Radiation therapy for pregnant women is limited as to the area and the timing of treatment due to concerns about fetal doses. The risks are different according to the developmental stage of the embryo or fetus. Even if the primary beam is not focused on the area of the fetus during radiation therapy, the body absorbs low doses that are difficult to predict outside the treatment area. The source of the dose outside of the radiation field is impacted by three major components: 1) treatment head leakage, 2) collimator system scattering, and 3) internal patient scattering. Advanced radiation techniques, such as intensity-modulated radiation therapy (IMRT), have higher scattered low doses than three-dimensional conformal radiation therapy (3DCRT) due to collimator scattering and head leakage [1]. Potential risks to the fetus by low doses are also unpredictable; however, a previous study reported that mental retardation and organ malformations of the fetus arise following exposure to a threshold dose of $0.5 \mathrm{~Gy}$ 
with a $50 \%$ risk of effects at $1 \mathrm{~Gy}$ [2]. Despite identification of such a threshold, it is important to ensure that the fetus receives as little radiation as possible. In addition, the main potential complications of radiation exposure in the growth phase of the fetus were mentioned, and in the embryonic stage, they were sensitive to $0.10 \mathrm{~Gy}$ of radiation or less [3]. Therefore, the radiation treatment region of a pregnant patient should be planned carefully to reduce the fetal dose to levels as low as reasonably achievable (ALARA). The concept of ALARA emphasizes the importance of minimum radiation time and maximum distance from the radiation field [4].

Tomotherapy is now widely used for treatment of head and neck (H\&N) cancers due to the advantages of conformal dose distribution of the radiation field and reduction of radiation dose to critical organs [5, 6]; however, this low dose over the whole area compared to Linear Accelerator (LINAC)-based IMRT causes concern for application to pregnant women. Also, the beam-on time and monitor unit (MU) of tomotherapy are higher than those of LINAC-based 3DCRT and IMRT. Tomotherapy has not been used for pregnant women with $H \& N$ cancer, although several studies reported the results of radiotherapy for these cancers in pregnant patients $[7,8]$.

In this study, we evaluated the dose absorbed by the fetus during tomotherapy for patients with H\&N cancer in comparison with that during 3DCRT without being shielded.

\section{Materials and Methods}

\section{Selection of the patient}

A 32-year-old woman who was 26 weeks pregnant with external auditory canal cancer received preoperative radio-

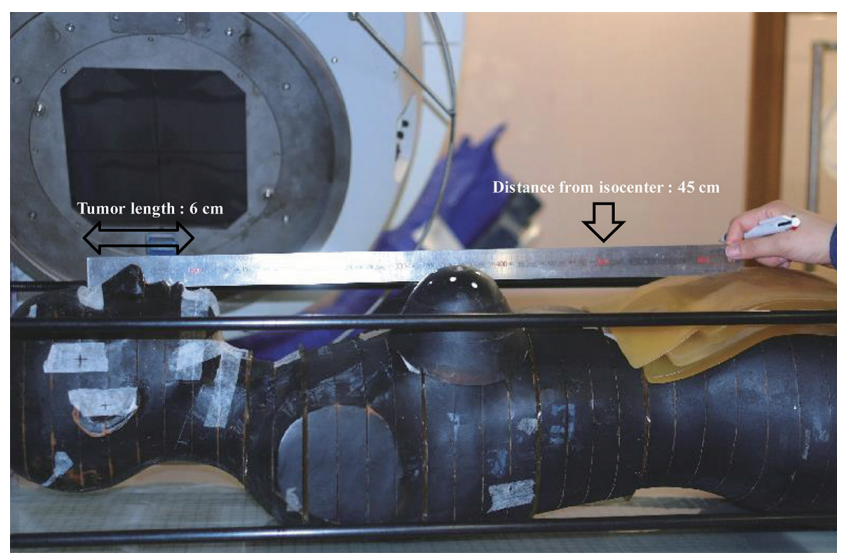

Fig. 1. Schematic diagram of the distance from the isocenter to the uterine fundus at 26 weeks of gestation. therapy without chemotherapy. The initial clinical stage was T2N0. We defined the planning target volume (PTV) and the clinical target volume (CTV) to include the entire external auditory canal plus a 1 to $2 \mathrm{~cm}$ margin, resulting in a maximum diameter of $6 \mathrm{~cm}$ for treatment. Prophylactic neck irradiation was not performed. We prescribed the dose to the CTV and PTV as 2.0 and $1.8 \mathrm{~Gy}$, respectively, in 20 fractions, and the volume of CTV and PTV were 31.2 and $84.9 \mathrm{~mL}$, respectively.

\section{In vitro measurement of phantom model}

For in vitro measurements, the patient was modeled using a humanoid phantom (Alderson Research Laboratory, Long Island, NY, USA). The geometry was estimated based on the growth of the fetus and the related position of the uterine fundus. The distance from the isocenter to the fundus was 45 $\mathrm{cm}$ in concordance with a gestation period of 26 weeks (Figure 1) [2]. The position of the uterine fundus was expressed by applying a bolus over the phantom surface. The dose for the treatment region included the neck around the target level (i.e., $10 \mathrm{~cm}$ from the isocenter) and both breast areas. In the abdomen, doses on the fundus and fetal region divided in the center, anterior, and posterior were measured.

\section{Tomotherapy versus 3DCRT}

Tomotherapy was provided with a TomoTherapy HiArt treatment machine (Tomotherapy, Madison, WI, USA) with $6 \mathrm{MV}$ and MLC of $0.625 \mathrm{~cm}$ widths. Tomol and Tomo2 plans were applied with field widths of 2.5 and $5 \mathrm{~cm}$ sized jaws, respectively, with a modulation factor of 2. The 3DCRT plan was carried out using a synergy linear accelerator equipped with a $1.0 \mathrm{~cm}$ MLC (Elekta, Synergy, Stockholm, Sweden). Three fixed beams of $6 \mathrm{MV}$ were used. Tomotherapy using 2.5 and $5 \mathrm{~cm}$ jaws and 3DCRT were delivered with 2,144, 1,372, and $301 \mathrm{MU}$, respectively (Table 1).

We preferentially measured the same set-up doses on the 3DCRT while all leaves were closed to detect leakage and scattering, which affects the fetal dose. The same 304 MU

Table 1. Treatment Planning Parameters for Tomo1, Tomo2, and 3DCRT

\begin{tabular}{lccc}
\hline & Tomo1 & Tomo2 & 3DCRT \\
\hline MLC (cm) & 0.625 & 0.625 & 1.000 \\
Remark & $2.5 \mathrm{~cm}$ jaw & $5 \mathrm{~cm}$ jaw & MLC \\
Total MU & 2,144 & 1,372 & 304 \\
Beam-on time (sec) & 160 & 113 & 30 \\
\hline
\end{tabular}



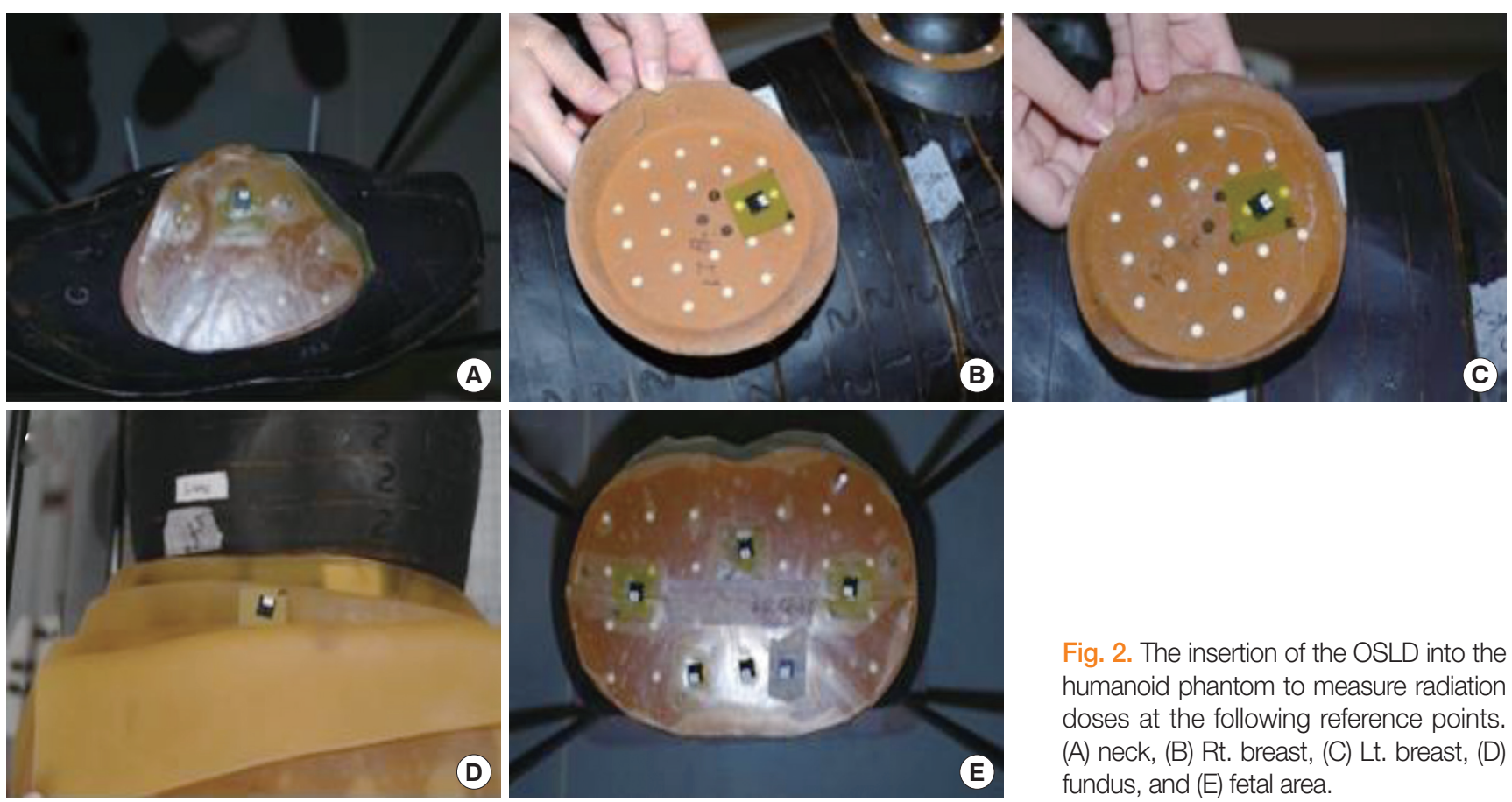

Fig. 2. The insertion of the OSLD into the humanoid phantom to measure radiation doses at the following reference points. (A) neck, (B) Rt. breast, (C) Lt. breast, (D) fundus, and (E) fetal area.

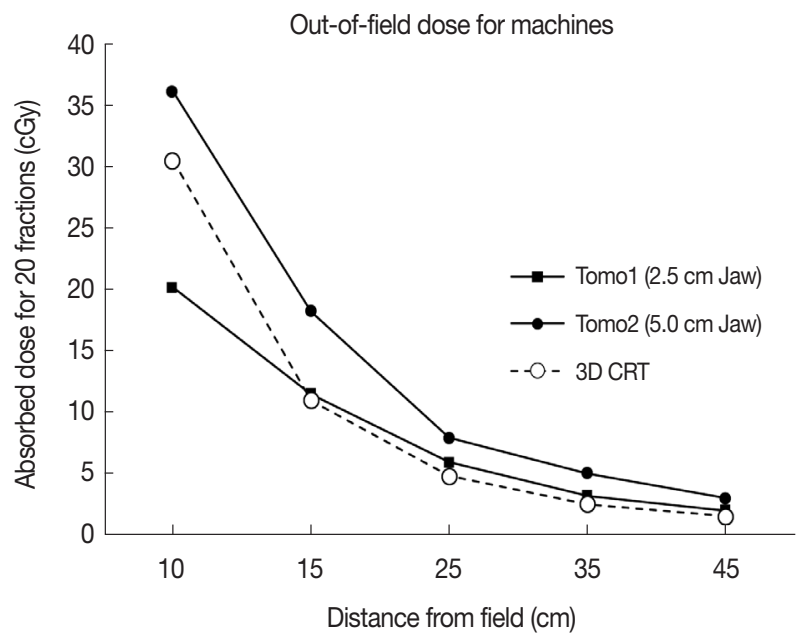

Fig. 3. Comparison of off-field doses for Tomo1, Tomo2, and 3DCRT according to the distances from the isocenter to the uterine fundus of the phantom.

was delivered. For comparison between tomotherapy and 3DCRT, the central dose at the fundus on each machine according to the distance from the target was measured. These fundus doses were compared between $45 \mathrm{~cm}$ at 26 weeks and $35 \mathrm{~cm}$ at 29 weeks. To compare the dose on treatment and fetus areas, we inserted groups of Optically Stimulated Luminescence Dosimeter (OSLD) between slices of the phantom and measured the dose at each position (Figure 2).
Table 2. Difference of Doses at Fundus between 26 and 29 Weeks of Gestation

\begin{tabular}{lccc}
\hline & 26 weeks $\left(45 \mathrm{~cm}^{\star}\right)$ & 29 weeks $\left(35 \mathrm{~cm}^{\star}\right)$ & Difference (\%) \\
\hline Tomo1 (cGy) & 2.0 & 2.7 & $0.7(35.00)$ \\
Tomo2 (cGy) & 2.3 & 3.9 & $1.6(69.57)$ \\
3DCRT (cGy) & 1.6 & 2.1 & $0.5(31.25)$ \\
\hline
\end{tabular}

${ }^{*}$ Distance from isocenter to fundus.

\section{Results and Discussion}

Radiation treatment for pregnant woman was performed from 26 to 29 weeks. During this period, the fundus is assumed to have a $10 \mathrm{~cm}$ displacement from 45 to $35 \mathrm{~cm}$ with a reference to the isocenter for conservative evaluation. When the out-of-field dose according to the distance of the 3DCRT was analyzed, the dose at $10 \mathrm{~cm}$ from the isocenter was measured as $30.5 \mathrm{cGy}$. The total dose at $25 \mathrm{~cm}$ from the isocenter was measured as $4.1 \mathrm{cGy}$, and the dose at the $45 \mathrm{~cm}$ fundus was measured at $1.7 \mathrm{cGy}$. Of the total $1.7 \mathrm{cGy}$ dose received by the fundus, leakage accounted for about $1.6 \mathrm{cGy}$.

Comparison of the dose as a function of the distance from the field for each method is shown in Figure 3. The out-offield dose of Tomo2 with a $5 \mathrm{~cm}$ jaw was the highest, while the dose of 3DCRT was the lowest at $15 \mathrm{~cm}$ from the isocenter. Doses at the uterine fundus according to different modalities were 1.7, 2.0, and 2.4 cGy for 3DCRT, Tomo1, and Tomo2, respectively. As shown in Table 2, the dose differenc- 
Table 3. Overall Dose Delivered to the Treatment and Fetus Regions

\begin{tabular}{lccc}
\hline & Tomo1 (cGy) & Tomo2 (cGy) & 3DCRT (cGy) \\
\hline Fundus & 2.008 & 2.432 & 1.701 \\
Fetus right (center) & 1.609 & 2.122 & 1.643 \\
Fetus left (center) & 2.166 & 2.568 & 1.517 \\
Fetus center (anterior) & 2.159 & 2.069 & 1.791 \\
Fetus center (posterior) & 1.635 & 2.396 & 1.198 \\
Fetus right (posterior) & 1.756 & 2.336 & 1.638 \\
Fetus left (posterior) & 1.992 & 2.246 & 1.435 \\
\hline
\end{tabular}

es from 26 to 29 weeks increased to $0.7,1.6$, and 0.5 cGy for Tomol, Tomo2, and 3DCRT, respectively. The Tomo2 plan showed an increased rate of $30 \%$ more than the other modalities. Table 3 and Figure 4 show the measured doses from the patient and the fetus region according to each modality. For the prescribed dose of $40 \mathrm{~Gy}$, the Tomo 2 plan delivered the highest dose of $36.13 \mathrm{~Gy}$, while Tomol delivered the lowest dose of 20.18 Gy to the neck. All modalities rapidly decreased to about $20 \%$ of the prescribed dose in the breast area, which was $25 \mathrm{~cm}$ away from the isocenter. Most of the fetus region received less than $5 \%$ of the prescribed dose, although the Tomo2 plan delivered a higher dose to the fetus region than the other modalities.

Among various cancers, $\mathrm{H} \& \mathrm{~N}$ cancer requires particular caution regarding the peripheral radiation dose, as this type of cancer requires both high doses and longer treatment periods than other cancers. We evaluated a 32-year-old pregnant woman with external auditory cancer and recommended tomotherapy as an appropriate treatment modality. Using a humanoid phantom, our study confirmed that both tomotherapy and 3DCRT have a low impact on the fetus, which received less than $5 \%$ of the prescribed patient dose. Tomotherapy delivers more peripheral radiation to the fetal region due to the 5-fold longer beam-on time compared with 3DCRT. When comparing tomotherapy regimens, however, the effect on fetal dose appears to be more influenced by collimator scattering based on field size and leakage dose by the MLC than by the beam-on times. In accord with these results, another study reported that the peripheral dose was not prominently increased by beam-on time but by the collimator and leakage scattering by a larger field size [1]. Our result also showed that the Tomol with a $2.5 \mathrm{~cm}$ jaw had higher MU than the Tomo2 with a $5.0 \mathrm{~cm}$ jaw, but fetus dose was higher in Tomo2. The 3DCRT measured dose also confirmed that leakage irradiation is the major component, accounting for about $85 \%$ of the $1.64 \mathrm{cGy}$ delivered to the fundus. It re-

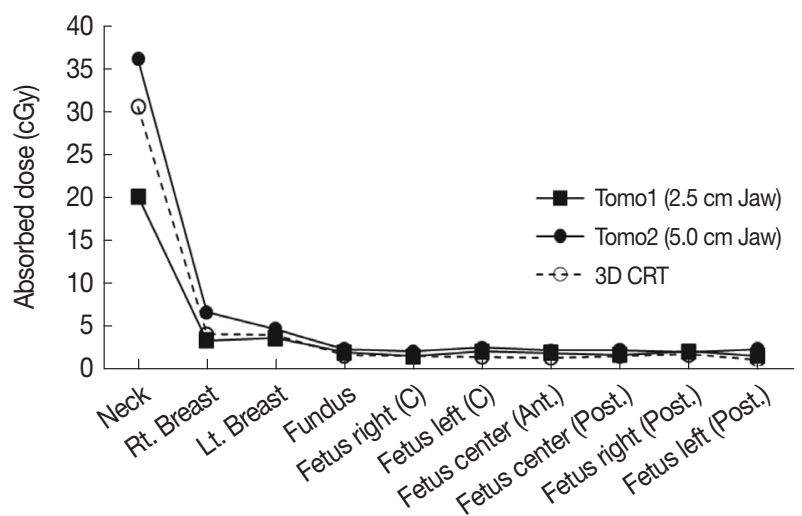

Fig. 4. Overall dose delivered to the treatment and fetus regions. The neck region is about $10 \mathrm{~cm}$ from the isocenter and is the treatment field margin point.

mains important, however, to analyze the effect of MU on peripheral dose. Because the radiation treatment plans represent different MU values, the peripheral irradiation dose and machine scattering are related to the MU [9]. Previous studies emphasized that the integral dose, which is affected by the out-of-field doses, is related to the MU, indicating that head leakage and collimator scatter increase as MU increases $[10,11]$.

From the target field, the radiation dose received by the fetus region was estimated to be about $5 \%$ of the prescribed dose of $40 \mathrm{~Gy}$. The dose decreased rapidly to about $20 \mathrm{~cm}$ or more from the isocenter (Table 3 and Figure 4). The peripheral dose was attenuated as the distance from the target increased, and the majority of the dose from the target results from leakage and scattering, as reported by Matthew B et al. [12]. These investigators showed that applying appropriate shielding for these peripheral doses can reduce the radiation doses to less than $50 \%$ of those without shielding on the $\mathrm{H} \& \mathrm{~N}$ cancer patient.

The radiation doses received by the fetus area were uniform in this study; however, the fetus position changes during radiation treatment as well as during gestation. Thus, it is important to consider the fetus position during the treatment period. Table 2 shows the dose effect on the fetus position; more doses of radiation were delivered to the same position after 3 additional weeks of gestation. Between 26 and 29 weeks, doses of Tomol ( $2.5 \mathrm{~cm}$ jaw), Tomo2 $(5 \mathrm{~cm}$ jaw), and 3DCRT increased $0.7 \mathrm{cGy}(35 \%), 1.6 \mathrm{cGy}$ (69.6\%), and 0.5 cGy (31.3\%), respectively. Dose increases by tomotherapy was proportional to jaw size. The dose at out-field of irradiation in the Tomo 2 plan was about $30 \%$ higher than that by 
3DCRT despite superior dose conformity. Moreover, the 5 $\mathrm{cm}$ jaw plan delivered $20 \%$ of the off-field dose compared to the $2.5 \mathrm{~cm}$ jaw plan, demonstrating the significant contribution of head scatter irradiation in tomotherapy. As a drawback, however, we need to measure the head leakage of tomotherapy although our preliminary study confirmed tomotherapy have different leakage dose according to the machine even in equal conditions.

This study did not evaluate post-shielding doses at different points of the fetus, but the results suggest appropriate shielding condition for each patient as the measured peripheral area doses were determined without shielding. In addition, image dose by scan range in the tomotherapy based on the image of megavoltage computed tomography (MVCT) is also important to the low dose regions. The further study is needed to measure in vivo dosimetry with actual patients using shielding conditions and acquiring the MVCT image to confirm the protection from radiation treatment.

\section{Conclusion}

In conclusion, we evaluated the radiation dose received by the fetus during preoperative radiotherapy for $\mathrm{H} \& \mathrm{~N}$ cancer. From the results of present study, we recommend tomotherapy with a $2.5 \mathrm{~cm}$ jaw for radiation treatment of pregnant patients with H\&N cancer. With 3DCRT, a major component of radiation dose is leakage irradiation, which is proportional to the MU. In tomotherapy, the fundus dose is highly dependent on head scattering, supporting a lower off-field dose with a smaller jaw size. Although tomotherapy is superior in normal tissue sparing, the fundus dose was about 20 to $30 \%$ higher than that with 3DCRT. Off-field irradiation is always dependent on dose, dose-volume, field size, and MU, and overall, the evaluation of risk of tomotherapy should be individually performed for each patient.

\section{Acknowledgements}

This research was supported by Basic Science Research Program through the National Research Foundation of Korea (NRF) funded by the Ministry of Education (NRF-2018 R1D1A1A0208572312).

\section{References}

1. Ruben JD, Lancaster CM, Jones P, Smith RL. A comparison of out-of-field dose and its constituent components for intensitymodulated radiation therapy versus conformal radiation therapy: implications for carcinogenesis. Int. J. Radiat. Oncol. Biol. Phys. 2011;81(5):1458-1464.

2. Prado KL, Nelson SJ, Nuyttens JJ, Williams TE, Vanek KN. Clinical implementation of the AAPM Task Group 36 recommendations on fetal dose from radiotherapy with photon beams: a head and neck irradiation case report. J. Appl. Clin. Med. Phys. 2000;1(1):1-7.

3. Sneed PK, Albright NW, Wara WM, Prados MD, Wilson CB. Fetal dose estimates for radiotherapy of brain tumors during pregnancy. Int. J. Radiat. Oncol. Biol. Phys. 1995;32(3):823-830.

4. Strauss KJ, Kaste SC. The ALARA (as low as reasonably achievable) concept in pediatric interventional and fluoroscopic imaging: striving to keep radiation doses as low as possible during fluoroscopy of pediatric patients--a white paper executive summary. Radiology. 2006;240(3):621-622.

5. Fiorino C, Dell'Oca I, Pierelli A, Broggi S, Martin ED, Muzio ND, Longobardi B, Fazio F, Calandrino R. Significant improvement in normal tissue sparing and target coverage for head and neck cancer by means of helical tomotherapy. Radiother. Oncol. 2006; 78(3):276-282.

6. van Vulpen M, Field C, Raaijmakers CP, Parliament MB, Tergaard CHJ, MacKenzie MA, Scrimger RS, Lagendijk JJW, Fallone BG. Comparing step-and-shoot IMRT with dynamic helical tomotherapy IMRT plans for head-and-neck cancer. Int. J. Radiat. Oncol. Biol. Phys. 2005;62(5):1535-1539.

7. Mazonakis M, Tzedakis A, Damilakis J. Monte Carlo Simulation of Radiotherapy for Breast Cancer in Pregnant Patients: How to Reduce the Radiation Dose and Risks to Fetus? Radiat. Prot. Dosimetry. 2017;175(1):10-16.

8. Mianji FA, Diba JK, Babakhani A. Fetus dose estimation in thyroid cancer post-surgical radioiodine therapy. Radiat. Prot. Dosimetry. 2015;163(1):27-36.

9. Hall EJ, Wuu CS. Radiation-induced second cancers: the impact of 3D-CRT and IMRT. Int. J. Radiat. Oncol. Biol. Phys. 2003;56(1): 83-88.

10. Fogliata A, Nicolini G, Alber M et al. IMRT for breast. a planning study. Radiother. Oncol. 2005;76(3):300-310.

11. Macklis R. In regards to Hall: intensity-modulated radiation therapy, protons, and the risk of second cancers (Int J Radiat Oncol Biol Phys 2006;65:1-7). Int. J. Radiat. Oncol. Biol. Phys. 2006;66(5): 1593-1594; author reply 5.

12. Podgorsak MB, Meiler RJ, Kowal H, Kishel SP, Orner JB. Technical management of a pregnant patient undergoing radiation therapy to the head and neck. Med. Dosim. 1999;24(2):121-128. 